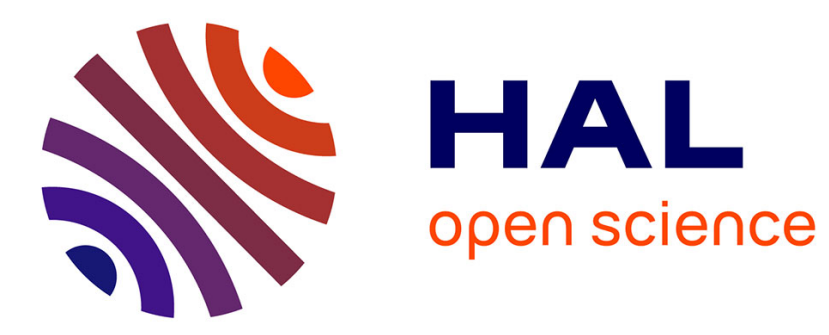

\title{
Frequency-domain ultrasonic NDE of three-layered media: the inverse problem
}

\author{
V. Kinra, C. Zhu
}

\section{To cite this version:}

V. Kinra, C. Zhu. Frequency-domain ultrasonic NDE of three-layered media: the inverse problem. Journal de Physique IV Proceedings, 1994, 04 (C5), pp.C5-1151-C5-1160. 10.1051/jp4:19945254 . jpa-00252943

\section{HAL Id: jpa-00252943 https://hal.science/jpa-00252943}

Submitted on 1 Jan 1994

HAL is a multi-disciplinary open access archive for the deposit and dissemination of scientific research documents, whether they are published or not. The documents may come from teaching and research institutions in France or abroad, or from public or private research centers.
L'archive ouverte pluridisciplinaire HAL, est destinée au dépôt et à la diffusion de documents scientifiques de niveau recherche, publiés ou non, émanant des établissements d'enseignement et de recherche français ou étrangers, des laboratoires publics ou privés. 


\title{
Frequency-domain ultrasonic NDE of three-layered media: the inverse problem
}

\author{
V.K. KINRA and C. ZHU \\ Center for Mechanics of Composites, Department of Aerospace Engineering, Texas A\&M University, \\ College Station, TX 77843, U.S.A.
}

\begin{abstract}
This paper presents a frequency-domain ultrasonic technique for a simultaneous determination of the thickness $(\mathrm{h})$ and wavespeed $(\mathrm{c})$ of the individual layers comprising a multilayered medium using the entire complex spectrum. Each of the layers may be "thin"; by thin we mean that the successive reflections of an ultrasonic pulse from the two faces of a layer are non-separable in the time domain. Plane longitudinal waves which are normally incident upon the medium are considered. A systematic analysis of the sensitivity of the complex-valued transfer function to the acoustical parameters of each of the layers has been carried out. Successful inversion results are presented for several three-layer specimens: aluminum/water/aluminum, aluminum/water/titanium, and titanium/water/titanium.
\end{abstract}

\section{INTRODUCTION}

There is a need for a technique for ultrasonic nondestructive evaluation (UNDE) of layered media in a variety of engineering applications: Our review of the literature revealed that a majority of the papers concerning multi-layered media are primarily concerned with the forward problem: Given the true values of the acoustical properties of the individual layer, compare the theory and experiment [1-6]. The purpose of this paper is to solve the inverse problem for a three-layered medium: Given a set of experimental results, estimate the unknown acoustical parameter using the entire complex Fourier spectrum. A systematic analysis of the sensitivity of the transfer function to the acoustical parameters of each of the layers has been carried out. An inverse algorithm has been developed to simultaneously reconstruct the thickness (h) and phase velocity (c) of each of the layers by minimizing the difference between the theory and the experiment in the mean-sum-square sense. Using this technique, the thickness and the wavespeed of the individual layers can be extracted from the same measurement, without knowing any of them. Results are presented for several three-layered specimens $\left(\mathrm{Al} / \mathrm{H}_{2} \mathrm{O} / \mathrm{Al}\right.$, $\mathrm{Al} / \mathrm{H}_{2} \mathrm{O} / \mathrm{Ti}$ and $\mathrm{Ti} / \mathrm{H}_{2} \mathrm{O} / \mathrm{Ti}$ ). Successful inversion was obtained for up to four parameters.

\section{THEORY}

Consider a three-layered specimen occupying the space $a<x<a+h_{1}+h_{2}+h_{3}$, immersed in an elastic fluid (water) and insonified by a time-harmonic longitudinal plane wave. We will use the subscript ()$_{1}$ to identify the immersion medium ()$_{0}$ and the layers ()$_{1},()_{2}$ and ()$_{3}$. An elementary application of the well-known Buckingham $\pi$ theorem [7] revealed that a wave "sees" a plate as a combination of the following three parameters: (1) the time taken by the wave to travel through one 
thickness of the plate, the transit time $\tau=\mathrm{h} / \mathrm{c}$; (2) the time taken by a wave travelling in the immersion medium (water) to travel through a distance $h$, the reference transit time $\gamma=h / c_{0}$; and (3) the acoustic impedance $\mathrm{Z}=\rho \mathrm{c}$, here $\rho$ is the density. Moreover, the Buckingham- $\pi$ theorem also discloses that the maximum number of $\pi$ groups required to completely describe the transfer function is three per plate. With these definitions, the transfer function defined in ref. [5] becomes

$$
\begin{gathered}
H^{*}(\omega)=\frac{16 Z_{0} Z_{1} Z_{2} Z_{3} e^{i \omega\left(\gamma_{1}+\gamma_{2}+\gamma_{3}+\tau_{1}+\tau_{2}+\tau_{3}\right)}}{\alpha_{0}\left(\alpha_{1} \alpha_{2}+\beta_{1} \beta_{2}\right)} \\
\alpha_{0}=\left(Z_{0}+Z_{1}\right)\left(Z_{1}+Z_{2}\right)\left(Z_{2}+Z_{3}\right)\left(Z_{3}+Z_{0}\right) \quad \alpha_{1}=e^{2 i \omega \tau_{1}}+\frac{\left(Z_{0}-Z_{1}\right)\left(Z_{1}-Z_{2}\right)}{\left(Z_{0}+Z_{1}\right)\left(Z_{1}+Z_{2}\right)} \\
\alpha_{2}=\left[e^{2 i \omega \tau_{3}}+\frac{\left(Z_{2}-Z_{3}\right)\left(Z_{3}-Z_{0}\right)}{\left(Z_{2}+Z_{3}\right)\left(Z_{3}+Z_{0}\right)}\right] e^{2 i \omega \tau_{2}} \\
\beta_{1}=\frac{Z_{1}-Z_{0}}{Z_{1}+Z_{0}}+\frac{Z_{1}-Z_{2}}{Z_{1}+Z_{2}} e^{2 i \omega \tau_{1}} \quad \beta_{2}=\frac{Z_{2}-Z_{3}}{Z_{2}+Z_{3}} e^{2 i \omega \tau_{3}}+\frac{Z_{3}-Z_{0}}{Z_{3}+Z_{0}}
\end{gathered}
$$

where $\omega$ is the circular frequency. In order to avoid confusion, from this point $h, c$, and $\rho$ will be referred to as the primary variables, while $\tau, \gamma$, and $\mathrm{Z}$ will be referred to as the $\pi$ variables.

\section{SENSITIVITY ANALYSIS}

Let $\mathrm{p}$ represent any of the acoustical parameters $(\tau, \gamma, \mathrm{Z})$ of a layered medium. A (complexvalued) sensitivity of the transfer function, $H^{*}(\omega)$, to $p$ is introduced as

$$
S_{H^{*}, p}=p \frac{\partial H^{*}(\omega)}{\partial p}
$$

Let $\delta_{\mathrm{p}}$ be the absolute error in estimating the parameter $\mathrm{p}$, let the normalized errors be defined as $\mathrm{e}_{\mathrm{H}}=\delta \mathrm{H} / \hat{\mathrm{H}}, \mathrm{e}_{\phi}=\delta \phi$, and $\mathrm{e}_{\mathrm{p}}=\delta \mathrm{p} / \hat{p}$, where the superscript (^) denotes a true value. For $\mathrm{j}=1,2,3, \ldots \mathrm{N}$, let $\omega_{\mathrm{j}}$ be $\mathrm{N}$ discrete FFT frequencies, then through a lengthy but straightforward calculation it can be shown that

$$
e_{p}=\frac{\sum_{j=1}^{N} H_{j}\left(S_{H, p}\right)_{j}\left(e_{H}\right)_{j}+H_{j}^{2}\left(S_{\phi, p}\right)_{j}\left(e_{\phi}\right)_{j}}{\sum_{j=1}^{N}\left(S_{p}\right)_{j}^{2}}
$$

where $S_{H, p}=\{p \partial H(\omega)\} /\{\partial p\}$ and $S_{\phi, p}=\{p \partial H(\omega)\} /\{\partial p\}$. Equation (3) establishes the "propagation of errors" from the measured quantity $\left(\mathrm{H}_{\mathrm{e}}^{*}\right)$ to the estimated parameters $\left(\vec{p}_{e}\right)$. Clearly, when the sensitivity is small, a small error in measuring $\mathrm{H}^{*}(\omega)$ will result in a large error in estimating $\mathrm{p}$, and vice versa. The sensitivities of $\mathrm{H}^{*}(\omega)$ to $\tau, \gamma$, and $\mathrm{Z}$ for the specimen $\mathrm{Z} 2 \mathrm{U}\left(\mathrm{Al} / \mathrm{H}_{2} \mathrm{O} / \mathrm{Ti}\right)$ are plotted in Figs. 1a-c over $0 \leq \mathrm{f} \leq 10 \mathrm{MHz}$ which includes the actual frequency range that was used for the inversion $(5.5 \leq f \leq 8.5$ $\mathrm{MHz}$ ). The corresponding sensitivity curves for the other two specimens may be found in [8]. We alert the reader that these sensitivities are not just functions of the material (intrinsic) properties; they depend strongly on the relative thicknesses (an extrinsic property) of the three plates which, for the present case, are: $\mathrm{Al} / \mathrm{H}_{2} \mathrm{O} / \mathrm{Ti}:$ : $2.022 / 0.495 / 0.876 \mathrm{~mm}$; see Table 1 . The following observations are made from these figures. The sensitivities to the properties of the water layer are very large. For the 
convenience of the reader we have plotted, along the abscissa, thickness/wavelength in the ith layer, $(\mathrm{h} / \lambda)_{\mathrm{i}} ;$ recall that $(\mathrm{h} / \lambda)_{\mathrm{i}}=0.5,1.0,1.5, \ldots$ correspond to the successive free-vibration resonant frequencies of the ith layer. It can be verified that the sensitivity to any parameter of any plate goes through a maximum when the structure goes through a resonance. Most importantly, with a collective reference to Fig. 1a-c, note that the sensitivity to the acoustic impedance is orders of magnitude smaller than the sensitivity to either the transit time or the reference transit time $\left(S_{\mathrm{Z}}<<\left(S_{\tau}, \mathrm{S} \gamma\right)\right)$. From equation (3), one can immediately conclude that the errors in estimating $\mathrm{Z}$ will be orders of magnitude larger than the errors in estimating $\tau$ or $\gamma$. From the design point of view, one should select a transducer whose bandwidth includes at least one - and preferably several - sensitivity peaks for each of the three plates under consideration.

\section{EXPERIMENTAL PROCEDURES}

All experiments were carried out in a water immersion apparatus in through-transmission mode. A schematic of the apparatus is shown in Fig.2. A detailed discussion of the measurement procedures may be found in [6]. Let $F^{*}(\omega)$ and $G^{*}(\omega)$ be, respectively, the Fourier Transforms of the incident wave and the transmitted wave, respectively. It has been shown [5] that $G^{*}(\omega) / F^{*}(\omega)=H^{*}(\omega)$. Here $H^{*}(\omega)$ is the transfer function given by equation (1). To improve the signal-to-noise ratio, each measurement of $H^{*}(\omega)$ was repeated 64 times and averaged. In this work, we tested several three-layer specimens: aluminum/water/aluminum, aluminum/water/titanium, and titanium/water/titanium. In assembling the three-layered specimens, aluminum spacers were used to form the water layer. The metallic plates $(2 \times 2$ in) and spacers were machined and polished such that the variation in thickness across the cross-section was less than $\pm 2.5 \mu \mathrm{m}$. The density of each specimen was determined using Archimedes' principle. The true wave speed of each of the constituent layers was determined using the magnitude spectrum [9]; the true thickness was determined using a micrometer. Table 1 lists the acoustical properties of the single plates in terms of the measured quantities (primary variables) $h, c$ and $\rho$.

\section{THE FORWARD PROBLEM}

Three three-layered specimens, $\mathrm{Z} 2 \mathrm{U}\left(\mathrm{Al} / \mathrm{H}_{2} \mathrm{O} / \mathrm{Ti}\right)$, E2C $\left(\mathrm{Al} / \mathrm{H}_{2} \mathrm{O} / \mathrm{Al}\right)$, and $\mathrm{U} 2 \mathrm{~T}\left(\mathrm{Ti} / \mathrm{H}_{2} \mathrm{O} / \mathrm{Ti}\right)$ were tested in this work. The measured and the predicted transfer functions for the case of specimen $\mathrm{Z} 2 \mathrm{U}$ are compared in Fig.3. Similar comparisons for specimens E2C and U2T can be found in [8]. The peaks in the amplitude spectrum correspond approximately to resonant frequencies of an individual layer. Away from the resonances the comparison is excellent; around the "water-peaks", the experimental magnitude is significantly less than the theoretical magnitude. It is conjectured that this discrepancy is due to the excitation of leaky Lamb waves which carry energy in the direction perpendicular to the direction of the incident longitudinal wave.

\section{TOPOGRAPHY OF THE ERROR SURFACE}

The essence of our inverse scheme is as follows: the best estimate of $(\vec{p})$ is the one which minimizes the difference between the theory and the experiment in the least-square sense, i.e., the one which minimizes the error function

$$
E(\bar{p})=\frac{1}{N} \sum_{j=1}^{N}\left\{\left\|H_{e}^{*}\left(\omega_{j}\right)-H^{*}\left(\omega_{j} ; \vec{p}\right)\right\|^{2}\right\}
$$

Note that here $H^{*}$ - and therefore $E$ - is viewed as a function of "variables" ( $\vec{p}$ ) $\Rightarrow$ $\left(\tau_{1}, \gamma_{1}, Z_{1}, \tau_{2}, \gamma_{2}, Z_{2}, \tau_{3}, \gamma_{3}, Z_{3}\right)$. In this section we simulate an "error-free perfect experiment" by replacing $\mathrm{H}_{\mathrm{e}}^{*}(\omega)$ with $\mathrm{H}^{*}(\omega)$ calculated at the true values of $(\vec{p})$, i.e., with "synthetic data". The objective of the inverse algorithm is to locate the true minimum of $E(\vec{p})$ in the $(\vec{p})$-space. We now present an elementary treatment of the relevant topography. Suppose we vary the parameters of only the first layer 
while holding the remaining six parameters fixed. A 3-D view and an accompanying contour plot of $\mathrm{E}\left(\gamma_{1}, Z_{1}\right)$ are shown in Fig.4a and $4 \mathrm{~b}$, respectively; similar plots for $\mathrm{E}\left(\tau_{1}, \mathrm{Z}_{1}\right)$ and $\mathrm{E}\left(\tau_{1}, \gamma_{1}\right)$ may be found in the remaining Fig.4. Perhaps the most striking feature of Fig.4a and $4 \mathrm{c}$ is that the slope in the $\mathrm{Z}_{1}$-direction is orders of magnitude smaller than the slope in the $\tau_{1}$ and $\gamma_{1}$-directions. In a real experiment (with an accuracy $e=0.1$ ), the inverse algorithm will converge to some point inside the contour $\mathrm{E}=\mathrm{e}^{2}=10^{-2}$; therefore, we will call it the error contour. From Fig. $4 \mathrm{~b}$, the approximate estimates of the error are: $\pm 1 \%$ in $\gamma_{1}$, and $> \pm 25 \%$ in $Z_{1}$; from Fig. $4 \mathrm{~d}: \pm 1 \%$ in $\tau_{1}$ and $> \pm 25 \%$ in $Z_{1}$; from Fig.4f: $\pm 1 \%$ in $\tau_{1}$ and $\pm 2 \%$ in $\gamma_{1}$. The error in estimating $Z_{1}$ is much larger than the error in estimating either $\tau_{1}$ or $\gamma_{1}$. This result is not altogether surprising in view of the fact that the sensitivity of $\mathbf{H}^{*}(\omega)$ to $\mathrm{Z}$ was noted to be orders of magnitude smaller than the sensitivity to $\tau$ and $\gamma$ (see Fig.3). With that we abandon the hope for measuring $\mathrm{Z}$ (this observation is not meant to be general but specific to the case in hand). and now view $\mathrm{E}$ as a function of the remaining six parameters $E(\bar{p})=E\left(\tau_{1}, \gamma_{1}, \tau_{2}, \gamma_{2}, \tau_{3}, \gamma_{3}\right)$. Contour plots of $E\left(\tau_{2}, \gamma_{2}\right)$ and $E\left(\tau_{3}, \gamma_{3}\right)$ are included in Fig.5a and $5 \mathbf{b}$, respectively. The estimates of the error are as follows: from Fig.5a: $\pm 0.5 \%$ in $\tau_{2}$ and $\pm 5 \%$ in $\gamma_{2}$; from Fig. $5 \mathrm{~b}: \pm 4 \%$ in $\tau_{3}$ and $\pm 3 \%$ in $\gamma_{3}$.

The attention is now turned to a three-parameter search $\left(\tau_{1}, \tau_{2}, \tau_{3}\right)$ holding $\left(\gamma_{1}, \gamma_{2}, \gamma_{3}\right)$ constant at their true values. Once again, the error contour corresponds to $\mathrm{E}=10^{-2}$. The error estimates are as follows (the contour plots are omitted for brevity): $-1 \%$ to $+0.5 \%$ in $\tau_{2}$ and $\pm 2 \%$ in $\tau_{1} ;-3 \%$ to $+6 \%$ in $\gamma_{1}$ and $\pm 1 \%$ in $\tau_{1}$; and $\pm 4 \%$ in $\tau_{3}$ and $\pm 1 \%$ in $\tau_{1}$. These estimates are in qualitative agreement with the sensitivity analysis (Fig. 1), where $S_{\tau_{2}}>S_{\tau_{1}}>S_{\tau_{3}}$.

\section{THE INVERSE PROBLEM}

The first step in the design of the inverse algorithm is to select a frequency range (and the corresponding transducers) based on the sensitivity analysis of eq.(2). For the $\mathrm{Al} / \mathrm{H}_{2} \mathrm{O} / \mathrm{Ti}$ specimen (Z2U) we selected $7.5 \mathrm{MHz}$ transducers with a useful bandwidth of $5.5 \leq \mathrm{f} \leq 8.5 \mathrm{MHz}$. This range includes five peaks of the sensitivity spectrum: two correspond to the resonances of the aluminum layer, two to the water layer, and one to the titanium layer (see also Figure 5a). A similar detailed sensitivity analysis was carried out for the other specimens (the corresponding figures may be found in [8]).

The computer code was written directly in terms of the desired parameters $h$ and $c$. First, an incremental search method (grid method) was used to locate the minimum value of the error function, eq. (4). Using the values returned by the incremental search method as the new current estimate, either the Simplex [9] or the Newton-Raphson method [10] was used to complete the search for the minimum. The search for the minimum was terminated when the successive iterations of the parameters, $\vec{p}$, agreed to within $10^{-4}$. It is important to note that - unlike sections I and II where synthetic data was used - now $E(\vec{p})$ is defined in terms of the real data. The presence of errors in $\mathrm{H}_{\mathrm{e}}^{*}$ has two effects on $E(\vec{p})$ : (1) The true minimum is shifted away from the true values of $\vec{p}$ to some point $\vec{p}_{e}$ (the estimated values) inside the error contour; and (2) The value of $E(\vec{p})$ at $\vec{p}=\vec{p}_{e}$ is greater than zero (the residual error). We were surprised by the fact that the convergence did not always occur at exactly the same values of $\vec{p}_{e}$. To understand this, we compared the error surface for the synthetic data and the real data on a magnified scale. In the case of the synthetic data, as expected, we observed only one minimum at $x=y=0$ (figure not included). In the case of the real data, this minimum "split" into two minima, which are very close to each other $( \pm 0.5 \%)$; Thus, the convergence may occur to either one of the these minima. This provides a qualitative answer to the question raised above. In all the tests conducted during this research, the inverse algorithm never converged to a false minimum.

The inversion succeeded for six cases: (1) $h_{1}, c_{1}$; (2) $h_{2}, c_{2}$; (3) $h_{3}, c_{3}$; (4) $h_{1}, h_{2}, h_{3}$;

(5) $c_{1}, c_{2}, c_{3} ;(6) h_{1}, h_{2}, h_{3}, c_{2}$. In Table 2 , we present the results of the mean value of a parameter (typical sample size $M=10$ ) and its precision (based on one standard deviation). The entry in the last column is calculated as follows 


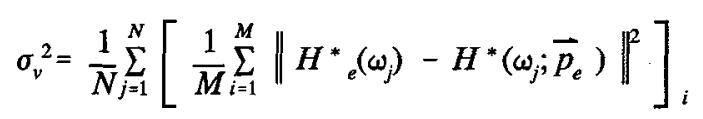

where $\vec{p}_{e}$ are the values of the parameters estimated (returned) by the inverse algorithm, and $M$ is the number of times the experiment was repeated. Thus $\sigma_{\mathrm{v}}$ is the residual error between the experimental $\mathrm{H}_{\mathrm{e}}^{*}$ and the theoretical $\mathrm{H}^{*}$ evaluated at $\vec{p}=\vec{p}_{e}$; in all cases $\sigma_{\mathrm{v}}<10 \%$. The precision in $\vec{p}_{e}$ is considered excellent: typically, $\pm 3 \mu \mathrm{m}$ in $\mathrm{h}$ and $\pm 0.1 \%$ in $\mathrm{c}$. The accuracy of $\vec{p}_{e}$ (the "true" values may be found in Table 1), which is not shown, is also considered excellent: it is about $\pm 2 \%$.

All of the work reported for the $\mathrm{Z} 2 \mathrm{U}\left(\mathrm{Al} / \mathrm{H}_{2} \mathrm{O} / \mathrm{Ti}\right)$ specimen - the forward problem, the sensitivity analysis, the topography of the error function, and the execution of the inverse algorithm was repeated for the two other specimens, namely, $\mathrm{E} 2 \mathrm{C}\left(\mathrm{Al} / \mathrm{H}_{2} \mathrm{O} / \mathrm{Al}\right)$ and $\mathrm{U} 2 \mathrm{~T}\left(\mathrm{Ti} / \mathrm{H}_{2} \mathrm{O} / \mathrm{Ti}\right)$. The details and the corresponding figures may be found in [8]. Only the final results are reported in Table $2 \mathrm{~b}$ for $\mathrm{E} 2 \mathrm{C}$, and $2 \mathrm{c}$ for $\mathrm{U} 2 \mathrm{~T}$. Essentially, these results are similar to those reported herein for the $\mathrm{Al} / \mathrm{H}_{2} \mathrm{O} / \mathrm{Ti}$ specimen.

\section{CONCLUSION}

A frequency-domain ultrasonic technique for a simultaneous determination of the thickness (h) and wavespeed (c) of the individual layers comprising a multi-layered medium is presented. Each of the layers may be thin. Plane longitudinal waves which are normally incident upon the medium are considered. A systematic analysis of the sensitivity of the complex-valued transfer function to the acoustical parameters has been carried out. The entire complex spectrum was used in the inverse problem. Using this technique, the thickness and the wavespeed of the individual layers can be extracted from the same measurement, without knowing any of them. Results are presented for several three-layer specimens: aluminum/water/aluminum, aluminum/water/titanium, and titanium/water/titanium. Successful inversion was obtained for the following cases: (1) simultaneous determination of $h$ and $c$ of any one of the three layers, given $h$ and $c$ of the remaining two layers; (2) simultaneous measurement of the three thicknesses, given the three wavespeeds; (3) simultaneous measurement of the three wavespeeds, given the three thicknesses; (4) simultaneous determination of all three thicknesses and one wavespeed, given the remaining two wavespeeds. The precision of our measurements was found to be extremely good: typically, $\pm 3 \mu \mathrm{m}$ in $\mathrm{h}$ and \pm one part per thousand in $c$. The accuracy was found to be about one order of magnitude worse than the precision: typically, $\pm 10 \mu \mathrm{m}$ in $\mathrm{h}$ and $\pm 2 \%$ in $\mathrm{c}$ (with the exception of the four-parameter inversion).

\section{ACKNOWLEDGEMENTS}

This work is based on the work supported by the Texas Advanced Research Program (Advanced Technology Program) under Grant No. 134 to Texas A\&M University, College Station, TX. Thanks are due to Professor John L. Junkins for providing us with many insights into the inverse problem , to Mr. Nathan Day for calculating the free-vibration resonant frequencies, and Ms. S.E. Hanneman for many helpful discussions.

\section{REFERENCES:}

1. Thomson, W.T., "Transmission of Elastic Waves Through a Stratified Solid Medium," J. of Appl. Phys., 21, pp.89-93 (1950).

2. Haskell, N.A., "The Dispersion of Surface Waves on Multilayered Media," Bulletin of the Seismological Soc. Am., 43, pp.17-34 (1953).

3. Folds, D.L. and Loggins, C.D, Transmission and reflection of ultrasonic waves in layered media, J. Acoust. Soc. Am., 62, pp.1102-1109 (1977).

4. Scott, W.R. and Gordon, P.F., Ultrasonic spectrum analysis for nondestructive 
testing of layered composite materials, J. Acoust. Soc. Am., 62, (1977) 108-116.

[5] Hanneman, S.E., and Kinra, V.K., "A New Technique for Ultrasonic Nondestructive Evaluation of Adhesive Joints: Part I. Theory," J. Exp. Mech., 32, (1992) 323-331.

[6] Hanneman, S.H., Kinra, V.K., and Zhu, C., "A New Technique for Ultrasonic Nondestructive Evaluation of Adhesive Joints: Part II. Experiment," J. Exp. Mech., 32, (1992) 332-339.

[7] White, F.M., Viscous Fluid Flow (McGraw-Hill Book Company, New York, 1974).

[8] Jaminet, P.T., "Ultrasonic Quantitative NDE of Layered Media: The Inverse Problem," Masters Thesis, Texas A\&M University, College Station, TX, December 1993.

[9] Walmsley, D.A., "The Simplex Method for Minimization of a General Function," (Transport and Road Research Laboratory, Crowthorne, Berkshire England, 1981, TRRL Supplementary Report No. 686).

[10] V. R. Iyer and V. K. Kinra, "Characterization of a Sub-Half-Wavelength Adhesive Layer Using Ultrasonic Spectroscopy," Enhancing Analysis Techniques for Composites Materials, Eds.: L. Schwer, J. N. Reddy and A. K. Mal (ASME NDE-Vol. 10, 1991) 35-42.
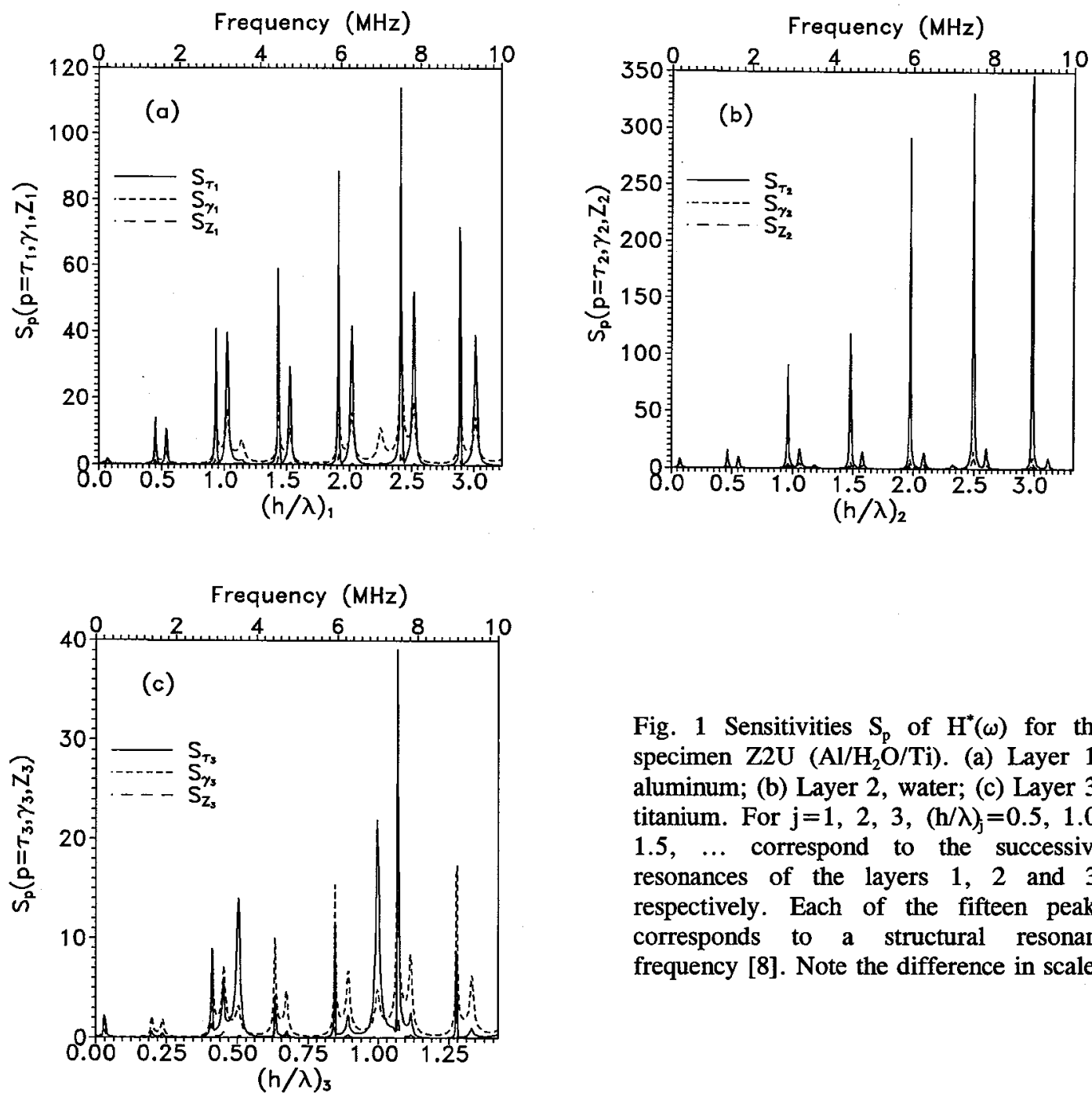

Fig. 1 Sensitivities $S_{p}$ of $H^{*}(\omega)$ for the specimen $\mathrm{Z} 2 \mathrm{U}\left(\mathrm{Al} / \mathrm{H}_{2} \mathrm{O} / \mathrm{Ti}\right)$. (a) Layer 1 , aluminum; (b) Layer 2, water; (c) Layer 3, titanium. For $\mathrm{j}=1,2,3,(\mathrm{~h} / \lambda)_{j}=0.5,1.0$, $1.5, \ldots$ correspond to the successive resonances of the layers 1,2 and 3 , respectively. Each of the fifteen peaks corresponds to a structural resonant frequency [8]. Note the difference in scale. 


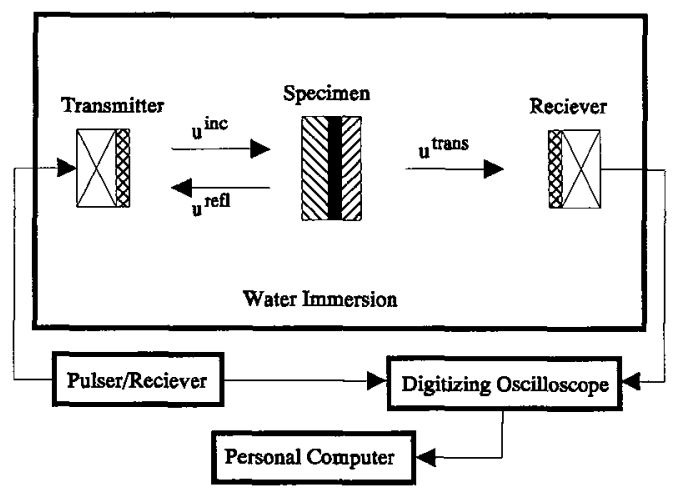

Figure $2 \mathrm{~A}$ schematic of the experimental setup.
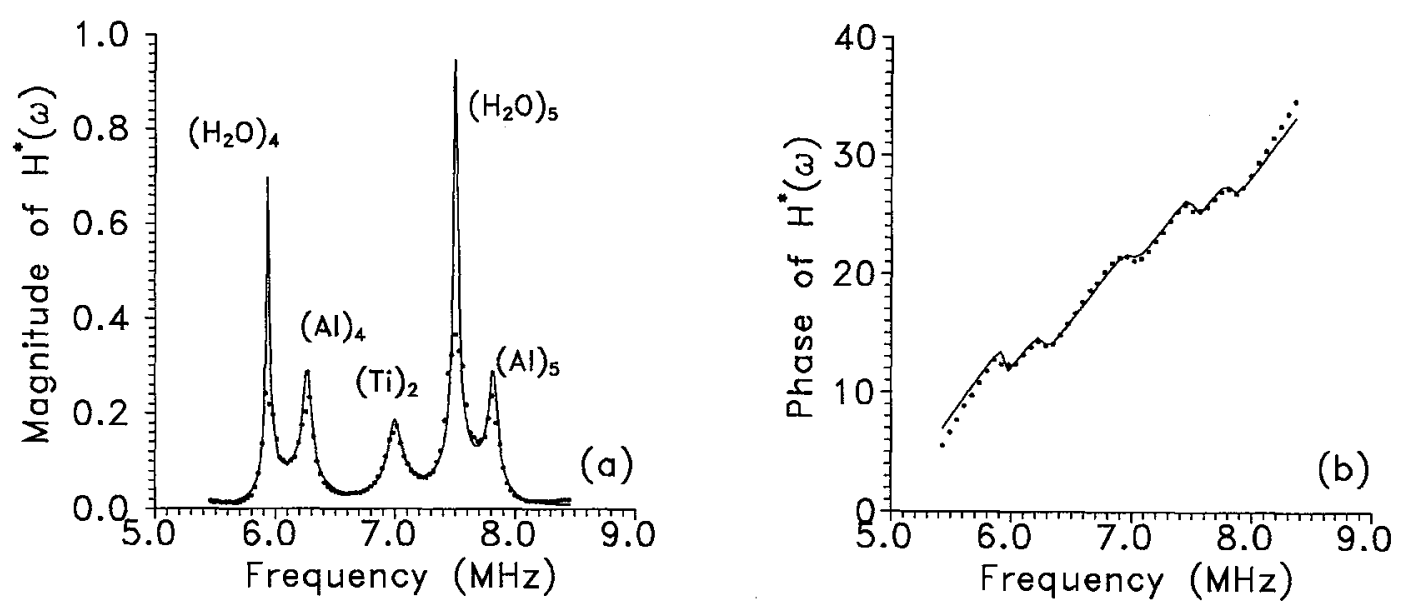

Fig.3 Forward comparison for specimen $\mathrm{Z} 2 \mathrm{U}\left(\mathrm{Al} / \mathrm{H}_{2} \mathrm{O} / \mathrm{Ti}\right)$. (a) The magnitude spectrum; (b) The phase spectrum.

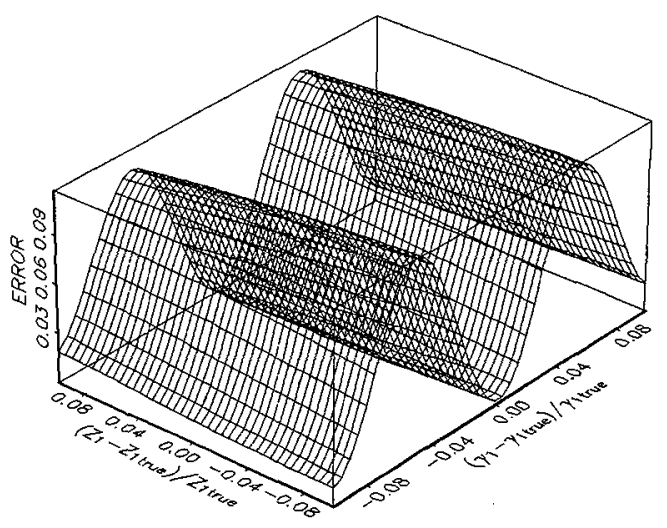

$4 a$

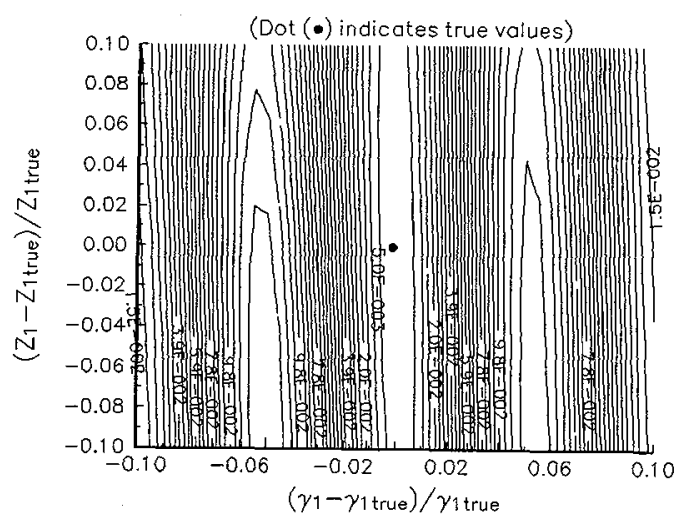

$4 b$ 


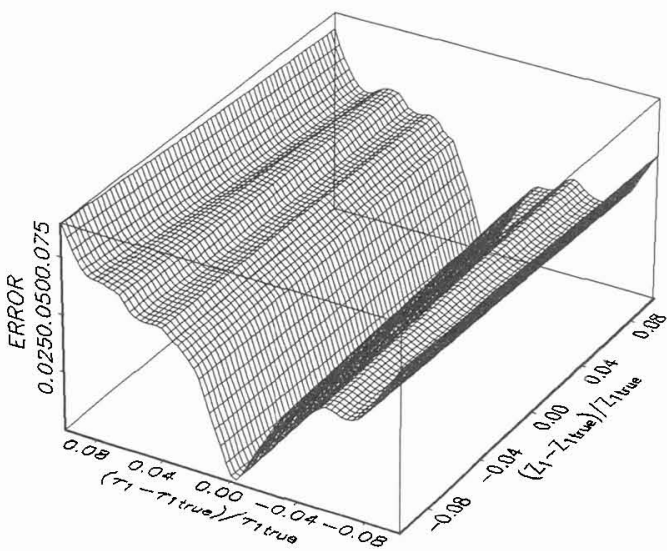

$4 \mathrm{c}$

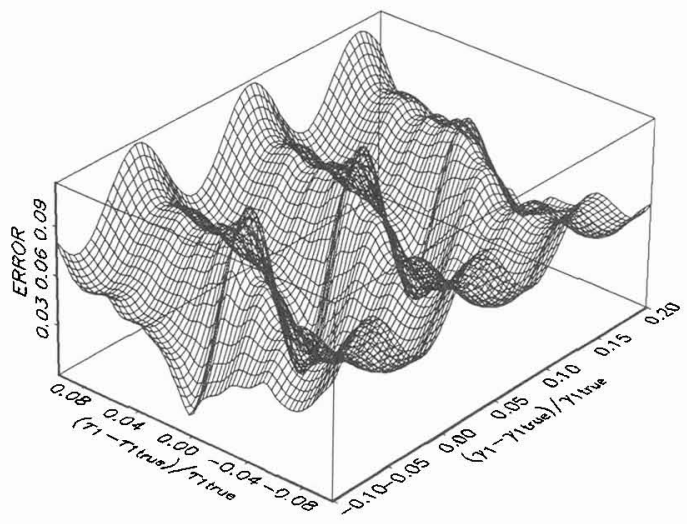

$4 \mathrm{e}$

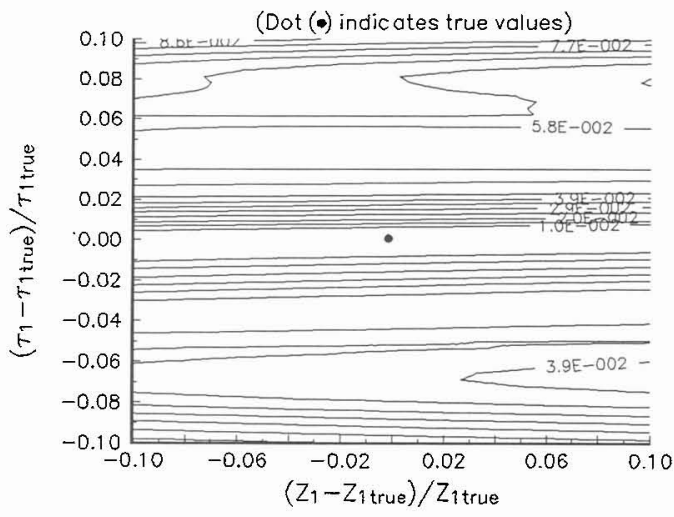

$4 d$

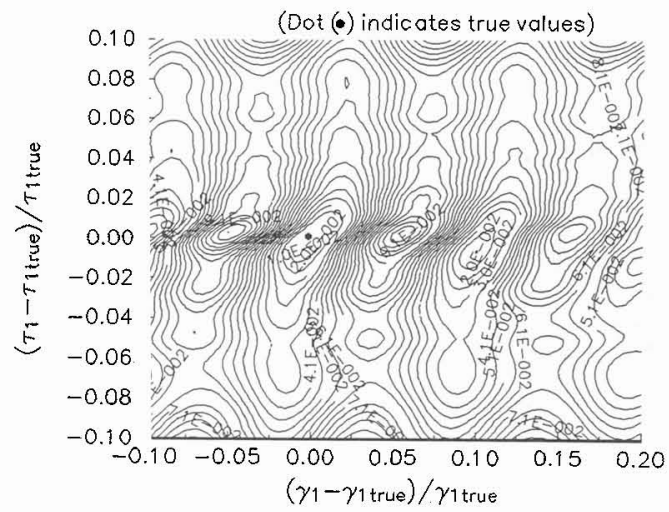

$4 \mathrm{f}$

Fig.4 Topography of the error surface for the specimen $\mathrm{Z} 2 \mathrm{U}\left(\mathrm{Al} / \mathrm{H}_{2} \mathrm{O} / \mathrm{Ti}\right)$. $\mathrm{E}\left(\gamma_{1}, \mathrm{Z}_{1}\right)$ : (a) and (b); $\mathrm{E}\left(\tau_{1}\right.$, $\left.Z_{1}\right):(c)$ and (d); and $E\left(\tau_{1}, \gamma_{1}\right):(e)$ and (f).

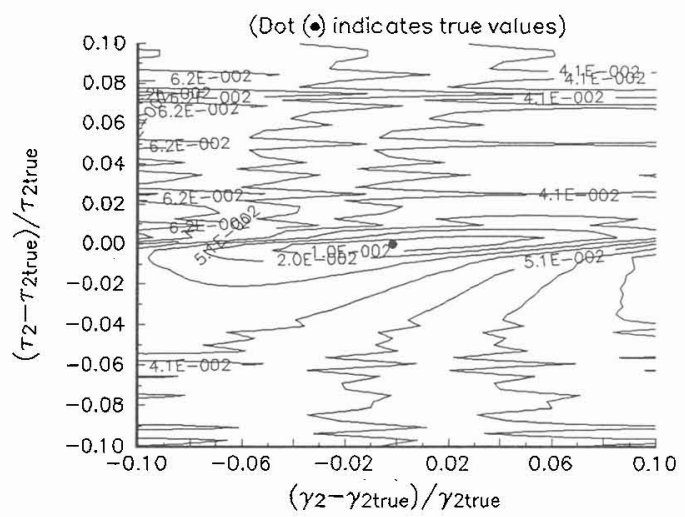

$5 a$

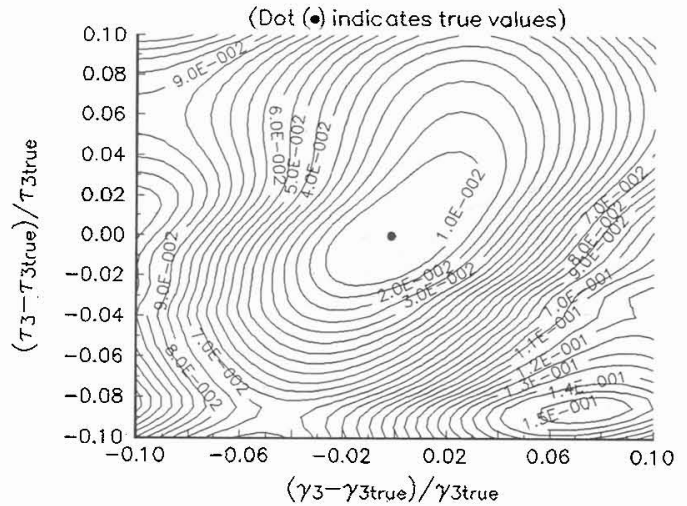

$5 \mathrm{~b}$

Fig.5 Topography of the error surface for the specimen $\mathrm{Z} 2 \mathrm{U}\left(\mathrm{Al} / \mathrm{H}_{2} \mathrm{O} / \mathrm{Ti}\right)$. Contour plot of (a) E vs. $\tau_{2}$ and $\gamma_{2} ;$ (b) E vs. $\tau_{3}$ and $\gamma_{3}$. 
Table 1: Acoustical Properties of the Constituents: Measured Quantities

\begin{tabular}{|c|c|c|c|}
\hline $\begin{array}{c}\text { Sample ID: } \\
(\text { Material })\end{array}$ & $\begin{array}{c}\text { Thickness } h(\mathrm{~mm}) \\
( \pm 0.0025 \mathrm{~mm})\end{array}$ & $\begin{array}{c}\text { Wavespeed } c(\mathrm{~mm} / \mu \mathrm{s}) \\
( \pm 0.03 \%)\end{array}$ & $\begin{array}{c}\text { Density } \rho\left(\mathrm{g} / \mathrm{mm}^{3}\right) \\
\left( \pm 0.0025 \mathrm{~g} / \mathrm{mm}^{3}\right)\end{array}$ \\
\hline C (Al) & 1.552 & 6.303 & $2.807 \mathrm{E}-2$ \\
\hline E (Al) & 1.334 & 6.287 & $2.804 \mathrm{E}-2$ \\
\hline Z (Al) & 2.022 & 6.278 & $2.809 \mathrm{E}-2$ \\
\hline T (Ti) & 0.876 & 6.160 & $4.518 \mathrm{E}-2$ \\
\hline U (Ti) & 0.876 & 6.160 & $4.518 \mathrm{E}-2$ \\
\hline Spacer 2 & 0.495 & 1.490 & $0.997 \mathrm{E}-2$ \\
\hline
\end{tabular}

Table 2a: Inversion Results for Specimen E2C $\left(\mathrm{Al} / \mathrm{H}_{2} \mathrm{O} / \mathrm{Al}\right):$ Mean Value \pm Precision

\begin{tabular}{|c|c|c|c|c|c|c|c|}
\hline \multicolumn{8}{|c|}{ Specimen Id: E2C } \\
\hline $\begin{array}{l}\text { Inversion } \\
\text { for: }\end{array}$ & $\begin{array}{c}\mathrm{h}_{1 \text { measured }} \\
(\mathrm{mm})\end{array}$ & $\begin{array}{l}\mathrm{c}_{1 \mathrm{~m} \text { tasured }} \\
(\mathrm{mm} / \mu \mathrm{s})\end{array}$ & $\begin{array}{c}\mathrm{h}_{2 \text { measured }} \\
(\mathrm{mm})\end{array}$ & $\begin{array}{c}\mathrm{c}_{2 \mathrm{measured}} \\
(\mathrm{mm} / \mu \mathrm{s})\end{array}$ & $\begin{array}{c}h_{3 \text { measured }} \\
(\mathrm{mm})\end{array}$ & $\begin{array}{c}\mathrm{c}_{3 \text { measured }} \\
(\mathrm{mm} / \mu \mathrm{s})\end{array}$ & $\sigma_{\mathrm{v}}$ \\
\hline $\mathrm{h}_{1}, \mathrm{c}_{1}$ & $1.320 \pm 0.001$ & $6.174 \pm 0.0005$ & --- & --- & --- & --- & 0.09 \\
\hline $\mathrm{h}_{2}, \mathrm{c}_{2}$ & $\cdots$ & --- & $0.501 \pm 0.001$ & $1.487 \pm 0.003$ & $\cdots$ & -- & 0.08 \\
\hline $\mathrm{h}_{3}, \mathrm{c}_{3}$ & --- & $-\cdots$ & $-\cdots$ & $-\cdots$ & $1.543 \pm 0.003$ & $6.167 \pm 0.007$ & 0.03 \\
\hline$c_{1}, c_{2}, c_{3}$ & $-\cdots$ & $6.277 \pm 0.008$ & $-\cdots$ & $1.480 \pm 0.002$ & --- & $6.218 \pm 0.004$ & 0.02 \\
\hline$h_{1}, h_{2}, h_{3}$ & $1.326 \pm 0.001$ & --- & $0.501 \pm 0.0003$ & --- & $1.563 \pm 0.001$ & $\cdots$ & 0.07 \\
\hline $\mathrm{h}_{1}, \mathrm{~h}_{2}, \mathrm{~h}_{3}, \mathrm{c}_{2}$ & $1.336 \pm 0.001$ & --- & $0.477 \pm 0.002$ & $1.422 \pm 0.004$ & $1.576 \pm 0.001$ & ---- & 0.06 \\
\hline
\end{tabular}


Table 2b: Inversion Results for Specimen $\mathrm{Z2U}\left(\mathrm{Al} / \mathrm{H}_{2} \mathrm{O} / \mathrm{Ti}\right):$ Mean Value \pm Precision

\begin{tabular}{|c|c|c|c|c|c|c|c|}
\hline \multicolumn{8}{|c|}{ Specimen Id: Z2U } \\
\hline $\begin{array}{l}\text { Inversion } \\
\text { for: }\end{array}$ & $\begin{array}{c}h_{\text {lmeasured }} \\
(\mathrm{mm})\end{array}$ & $\begin{array}{l}c_{1 \text { measured }} \\
(\mathrm{mm} / \mu \mathrm{s})\end{array}$ & $\begin{array}{c}\mathbf{h}_{2 \text { measured }} \\
(\mathrm{mm})\end{array}$ & $\begin{array}{c}c_{2 m e a s u r e d} \\
(\mathrm{~mm} / \mu \mathrm{s})\end{array}$ & $\begin{array}{c}\mathrm{h}_{3 \text { measured }} \\
(\mathrm{mm})\end{array}$ & $\begin{array}{c}\mathrm{c}_{3 \mathrm{measured}} \\
(\mathrm{mm} / \mu \mathrm{s})\end{array}$ & $\sigma_{\mathrm{v}}$ \\
\hline $\mathrm{h}_{1}, \mathrm{c}_{1}$ & $2.001 \pm 0.001$ & $6.257 \pm 0.004$ & $\cdots$ & $\cdots$ & --- & $-\cdots$ & 0.09 \\
\hline $\mathrm{h}_{2}, \mathrm{c}_{2}$ & --- & --- & $0.503 \pm 0.001$ & $1.503 \pm 0.003$ & --- & $-\ldots$ & 0.08 \\
\hline $\mathrm{h}_{3}, \mathrm{c}_{3}$ & --- & --- & --- & -- & $0.861 \pm 0.009$ & $6.077 \pm 0.046$ & 0.09 \\
\hline $\mathrm{c}_{1}, \mathrm{c}_{2}, \mathrm{c}_{3}$ & --- & $6.288 \pm 0.002$ & $-\cdots$ & $1.479 \pm 0.0002$ & $\cdots$ & $6.166 \pm 0.003$ & 0.08 \\
\hline $\mathrm{h}_{1}, \mathrm{~h}_{2}, \mathrm{~h}_{3}$ & $2.022 \pm 0.005$ & $-\cdots$ & $0.478 \pm 0.002$ & --- & $0.877 \pm 0.004$ & --- & 0.08 \\
\hline $\mathrm{h}_{1}, \mathrm{~h}_{2}, \mathrm{~h}_{3}, \mathrm{c}_{2}$ & $2.023 \pm 0.0004$ & --- & $0.502 \pm 0.001$ & $1.500 \pm 0.003$ & $0.878 \pm 0.0003$ & $\cdots$ & 0.08 \\
\hline
\end{tabular}

Table 2c: Inversion Results for Specimen U2T $\left(\mathrm{Ti} / \mathrm{H}_{2} \mathrm{O} / \mathrm{Ti}\right):$ Mean Value \pm Precision

\begin{tabular}{|c|c|c|c|c|c|c|c|}
\hline $\begin{array}{l}\text { Inversion } \\
\text { for: }\end{array}$ & $\begin{array}{c}\mathbf{h}_{1 \text { measured }} \\
(\mathrm{mm})\end{array}$ & $\begin{array}{l}c_{1 \text { measured }} \\
(\mathrm{mm} / \mu \mathrm{s})\end{array}$ & $\begin{array}{c}\mathrm{h}_{\text {2measured }} \\
(\mathrm{mm})\end{array}$ & $\begin{array}{c}\mathrm{c}_{2 \mathrm{measured}} \\
(\mathrm{mm} / \mu \mathrm{s})\end{array}$ & $\begin{array}{c}\mathrm{h}_{3 \text { measured }} \\
(\mathrm{mm})\end{array}$ & $\begin{array}{l}c_{3 \text { measured }} \\
(\mathrm{mm} / \mu \mathrm{s})\end{array}$ & $\sigma_{\mathrm{v}}$ \\
\hline$h_{1}, c_{1}$ & $0.864 \pm .001$ & $6.071 \pm 0.007$ & ---- & $\therefore-$ & ---- & $\cdots$ & 0.17 \\
\hline $\mathrm{h}_{2}, \mathrm{c}_{2}$ & -- & $\cdots$ & $0.4713 \pm 0.003$ & $1.438 \pm 0.005$ & --- & -- & 0.07 \\
\hline $\mathrm{h}_{3}, \mathrm{c}_{3}$ & --- & $--\cdot$ & --- & $\cdots$ & $0.864 \pm 0.001$ & $6.072 \pm 0.007$ & 0.05 \\
\hline $\mathrm{h}_{1}, \mathrm{~h}_{2}, \mathrm{~h}_{3}$ & $0.879 \pm 0.003$ & $\cdots$ & $0.493 \pm 0.003$ & $\cdots$ & $0.881 \pm 0.003$ & $=$ & 0.14 \\
\hline $\mathrm{h}_{1}, \mathrm{~h}_{2}, \mathrm{~h}_{3}, \mathrm{c}_{2}$ & $0.878 \pm 0.003$ & --- & $0.480 \pm 0.001$ & $1.456 \pm 0.002$ & $0.877 \pm 0.003$ & -... & 0.09 \\
\hline
\end{tabular}

